\title{
Cervical cancer, geographical inequalities, prevention and barriers in resource depleted countries (Review)
}

\author{
ANNA BOGDANOVA, CHARLES ANDRAWOS and CONSTANTINA CONSTANTINOU \\ Department of Basic and Clinical Sciences, University of Nicosia Medical School, CY-1700 Nicosia, Republic of Cyprus
}

Received November 30, 2021; Accepted January 25, 2022

DOI: 10.3892/ol.2022.13233

\begin{abstract}
Cervical cancer (CC) remains a current global issue, with $>90 \%$ of cervical cancer cases being attributed to human papilloma virus (HPV). The highest burden of cervical cancer is reported in resource-depleted geographical areas with a high incidence of HPV infection. Recent developments in primary prevention include vaccinations against specific strains of HPV and the psychoeducation of the public. Yet, despite the availability of vaccinations, there is high incidence of both HPV and cervical cancer in developing countries, which is attributed to a multitude of barriers including inaccessibility to expensive vaccines. With regards to secondary prevention, progress is actively being made to develop more effective methods of screening and to specifically address the needs of low-income countries. In the past few years, more novel screening methods, such as self-assessment kits, immunohistochemistry and methylation marker analysis, have been developed. Barriers to screening in resource-depleted countries include limited financial resources and infrastructure to develop screening programmes, a lack of screening programmes that are accessible to populations, inadequate training of healthcare teams and stigma related to medical examinations performed as part of screening. Developing primary and secondary prevention programs, as well as addressing the barriers involved in countries with low socioeconomic levels, can drastically reduce morbidity and mortality rates associated with cervical cancer, thus reducing the burden associated with this gynaecological malignancy.
\end{abstract}

Correspondence to: Dr Constantina Constantinou, Department of Basic and Clinical Sciences, University of Nicosia Medical School, 21 Ilia Papakyriakou, 2414 Engomi, P.O. Box 24005, CY-1700 Nicosia, Republic of Cyprus

E-mail: constantinou.co@unic.ac.cy

Key words: cervical cancer, primary prevention, psychoeducation, vaccinations, screening, methylation markers, human papilloma virus self-sampling, barriers

\section{Contents}

1. Introduction

2. Epidemiology and geographical inequalities in the burden of $\mathrm{CC}$

3. Primary prevention and barriers faced by developing countries

4. Vaccinations

5. Psychoeducation

6. Secondary prevention and challenges faced by developing countries

7. Recent developments in screening methods

8. Barriers to screening in low resource countries and strategies for improvement

9. Conclusion

\section{Introduction}

Cervical cancer (CC) is the fourth most common cancer in women. According to Globacon, in 2020, CC affected 604,127 patients and was responsible for 341,831 deaths (1). This disease typically occurs in sexually active females aged 30-45, but also presents beyond this age range. A number of well-established risk factors include a positive human papilloma virus (HPV) test, multiple sexual partners, smoking, early coitarche, lack of screening and immunosuppression $(2,3)$.

In the microenvironment of the cervix, a junction of columnar epithelium representing the endocervix and squamous epithelium representing the exocervix exists which is known as the transformation zone and remains the most susceptible zone to neoplastic proliferation in the cervix (4). HPV contributes to, but it is not the sole contributor to the development of CC. In fact, only approximately 5\% of HPV infected individuals develop carcinoma yet more than $90 \%$ of all cases of $\mathrm{CC}$ are attributed to $\operatorname{HPV}(5,6)$. It should also be noted that not all HPV strains inevitably give rise to cancer. HPV strains 6 and 11 may cause warts and papillomatosis of the respiratory tract. HPV strains $-16,-18,-31,-33,-45,-52,-58$ on the other hand are associated with carcinogenesis of the cervix (7). HPV-16 and HPV-18 produce the E6 and E7 gene products, which in turn inhibit the $\mathrm{p} 53$ and $\mathrm{Rb}$ proteins respectively (8).

$\mathrm{CC}$ is often asymptomatic, allowing time for it to progress to a more invasive malignancy. With the gradual progression 
of the cancer, some symptoms emerge which include among others abnormal vaginal bleeding, dyspareunia, abnormal discharge, haematuria, urinary incontinence, lower abdominal pain and general fatigue $(9,10)$. The diagnosis for $\mathrm{CC}$ involves initial screening with a Papanicolaou (Pap) smear/cytology which has $55 \%$ sensitivity for CINII and $97 \%$ sensitivity for CINIII. If an abnormality is detected on Pap smear, this is followed up by colposcopy with biopsy, which is considered to be the gold standard for definitive diagnosis. The Pap test demonstrates low sensitivity for initial dysplastic events such as CINI, and therefore some physicians may opt for colposcopy with biopsy after a positive HPV test (11). Histological examination of a cervical biopsy categorises $\mathrm{CC}$ into two types of carcinomas: squamous cell carcinoma, which constitutes up to $85 \%$ of cancer samples and adenocarcinoma which reaches up to $25 \%$ of cases in certain areas (12). CC is typically preceded by cervical intraepithelial neoplasia (CIN) I-III, which can also be visualised on histology (13).

The staging system employed in CC follows The International Federation of Gynaecology and Obstetrics (FIGO) system and the management of $\mathrm{CC}$ is dependent on the staging of the tumour based on the FIGO system (14). For IA cervical cancer, the preferred mode of treatment is conisation which may include cold knife conisation, loop excision conisation or laser (loop excision is usually the treatment of choice at this stage) (15). Regarding stages IA2-IB2, a more radical approach is taken, involving hysterectomy, bilateral salpingectomy as well as possible bilateral oophorectomy and pelvic lymphadenectomy (16). For stages IB3-IVA, management includes external beam radiotherapy (EBRT), intracavitary brachytherapy and concomitant cisplatin chemotherapy.

Studies have shown that there is an unequal distribution of $\mathrm{CC}$ among different parts of the world as well as based on ethnic background $(17,18)$. This inequality may be due to a variety of factors such as lack of education related to both HPV and CC, lack of screening, genetic predisposition as well as exposure to more risk factors or cultural beliefs in certain geographical regions; yet probably one of the most important factors for a higher mortality rate in underdeveloped countries vs. developed countries is the lack of implementation of primary and secondary prevention programmes.

The aims of this narrative review are to examine geographical differences in the epidemiology of $\mathrm{CC}$, provide an overview of the most effective primary and secondary preventive measures available, describe the barriers existing in resource depleted countries (at the health system, the community and the individual levels) and describe the possible strategies that can be developed to further improve prevention and diminish the impact of this gynaecological malignancy.

\section{Epidemiology and geographical inequalities in the burden of $\mathrm{CC}$}

The global age-standardised incidence for cervical cancer for the year 2020 stands at 11.1 per 100,000 women while the age-standardised mortality rates is 14.8 per 100,000 women, with substantial variations from country to country (19). It is pertinent to note that while the worldwide prevalence of $\mathrm{CC}$ was reported as 604,127 cases in the year $2020,85 \%$ of the cases were reported in low- and middle-income countries (20).
The general trend elicited is that the higher the Human Development Index (HDI), the lower the incidence and mortality rates of CC. The HDI is a measure of a country's successes based on a range of social and economic factors. A value of 0 indicates poor performance, while a value of 1 indicates extremely high development. The main components of this index lie in the public health of the nation, education attainability and living standards (21). Some examples which highlight this HDI based inequality are Malawi, Zambia, Zimbabwe and Tanzania which reported an astonishing incidence of over 40 per 100,000-woman years as well as an HDI below 0.5 in 2018 . In the same year, approximately $84 \%$ of CC cases and $88 \%$ of CC related deaths occurred in countries with an HDI below 0.8. Malawi and Eswatini both have an HDI below 0.65 and exhibited an incidence of 72 and 75 per 100,000-women years in 2018 respectively. Meanwhile China and India have their HDIs fluctuating between 0.65 and 0.8 and demonstrated an incidence of 11 and 15 per 100,000-woman years respectively in the same year. These numbers can be immediately contrasted with a high HDI country such as Germany (0.95), where CC incidence stood at roughly 8 per 100,000-woman years. Interestingly, China and India together contributed towards $35 \%$ of the disease burden worldwide. At the same time, countries with a high HDI over 0.8 , such as Australia, New Zealand and Finland, exhibited a 2-4-fold lower incidence and mortality standing roughly at 7 and 3 per 100,000-woman years respectively when examined during the same year (22).

If trends are to be examined, high HDI countries such as Switzerland exhibited a decrease in CC incidence, from $0.98 \%$ in $1993-1997$ to $0.37 \%$ in 2008-2012 (23). The initial incidence was already relatively low, but over the years it decreased further. Looking at middle to high HDI countries such as Singapore, a larger drop can be observed: the incidence experienced a 9.5-fold decrease from 44.8 per 100,000-person years in 1968 to 4.7 per 100,000 -person years in 2012 in ethnically Indian women residing in Singapore. It is also remarkable that during this time period the decrease in incidence per 100,000-person years did not even plateau (24). Unfortunately, the trend differs for low HDI countries such as Uruguay. In the latter, there was a shallow decline in the incidence of $\mathrm{CC}$ from 17.6 to 14.8 per 100,000 -person years in the period of 2002-2015. One could argue that such a drastic difference in incidence reduction per person years between the Singaporean subpopulation and Uruguay is due to examining a larger time frame in the former study, so it would be unfair to draw conclusions about Uruguay since the study was shorter. This however is a false assumption since the decrease in incidence of CC for the Singaporean population between 2005 and 2010 was $50 \%$ (from 10 to 5 per 100,000-person years), while the decrease in the incidence in $\mathrm{CC}$ in the Uruguay population mentioned above was $16 \%$ during the period of 2002 and 2015. Hence, in a high HDI country there was a larger incidence reduction of CC during a period of 5 years compared to a country with low HDI country during a period of 13 years (25). The plateau in incidence for Uruguay and other low HDI countries, despite globalisation of HPV vaccinations and updated screening protocols, warrant a discussion regarding geographical inequalities.

Studies have shown that there is an unequal distribution of CC among different parts of the world also based on 
ethnic background. A study conducted during the period of 1999 to 2015 has shown that African-American women have substantially higher incidence $(10.8$ per 100,000$)$ and mortality $(4.4$ per 100,000$)$ rates than White American women (7.4 per 100,$000 ; 2.1$ per 100,000 respectively) (26). This is despite both groups of women residing in the Untited States of America, where screening and vaccination programs are in place. In comparison, rates in Sub-Saharan Africa are even more dismal: $\mathrm{CC}$ constituted the number one cause of death among women with a mortality rate of 30 per 100,000 for the year 2018 (27). This inequality may be due to a variety of factors such as lack of education in the field, genetics, exposure to more risk factors or cultural beliefs in certain geographical regions; yet probably one of the most important factors for a higher mortality rate in women in Sub-Saharan Africa compared to Caucasian women residing in first world countries is the lack of a stable implemented screening program or inaccessibility to such a programme in the former.

The worldwide prevalence of HPV stands at $12 \%$, while the prevalence in regions such as sub-Saharan Africa reaches $24 \%$. According to WHO, HPV infection with genotypes 16 and 18 is a direct cause of $70 \%$ of CC cases. In low HDI countries such as Ethiopia (0.49), a total of $90.2 \%$ of CC cases are attributed to HPV 16 and 18, 20\% higher than the global average (28). This raises the issue of lack of vaccination against HPV strains in such geographical areas, which could have reduced the incidence of $\mathrm{CC}$.

\section{Primary prevention and barriers faced by developing countries}

The purpose of primary prevention is to prevent a disease from ever occurring. Thus, primary prevention targets healthy individuals and focuses on interventions that are performed before development of a condition and include psychoeducation and vaccination (29). The European guidelines offer randomized control trial (RCT) and expert opinion supported recommendations for primary prevention, with the focus on HPV vaccination. The guidelines state that HPV vaccinations are best implemented through largescale national programmes, with the target age for vaccination being 10-13 years of age. Monitoring and evaluation of the safety and effectiveness of the HPV vaccine should also be implemented in for long term evaluation and hence improvement of the vaccination programme in each country. The coverage target was set at a minimum of $70 \%$ with a strong preference for over $80 \%$. Lastly, it was recommended that European countries focus on synergising HPV screening and vaccination as a potential method for lowering the financial burden of HPV related disease (30).

\section{Vaccinations}

The most effective method for CC primary prevention is HPV vaccination. The Food and Drug Administration has approved three different vaccines Cervarix, Gardasil and Gardasil 9 (31). Cervarix is a bivalent vaccine that covers HPV 16 and 18, the two most commonly involved strains in CC pathogenesis. Gardasil on the other hand is quadrivalent, covering for HPV 6, 11, 16 and 18. In the past few years,
HPV 31 and 33 have emerged as important types with higher predictive values than HPV18 in causing CC. Specifically, studies conducted in New Mexico and Sweden have shown that HPV 31 and 33 have a higher positive predictive value for CIN2+ and CIN3+ compared to HPV18. HPV 39, 56, 59, 66 and 68 have been shown to have an 'intermediate risk' and HPV 66 has been shown to carry very little risk. Lastly, Gardasil 9, offers a more extensive spectrum of coverage with the addition of HPV 31, 33, 45, 52 and 58 to the list of strains. Vaccination does not only protect from HPV derived $\mathrm{CC}$, but also yields protection from pre-cancerous and cancerous lesions of the lower genital tract, anal area, head and neck, as well as prevention of genital warts (32). Furthermore, studies proposed that even late vaccination provides a better population-based protection against HPV as opposed to no vaccination (33). Therefore, efforts should be invested to further develop vaccines that will cover a greater range of HPV strains involved in cervical carcinogenesis (34).

When vaccination levels are compared across countries there is a clear discrepancy seen between high HDI and low HDI countries. A study estimated that in 2019, New Zealand and Australia vaccinated $86 \%$ of females with the first dose and $77 \%$ with the second dose. During the same year, Sub Saharan Africa as a whole vaccinated 31 and $20 \%$ of females with the first and second doses respectively. The study also highlighted that during the same year higher HDI countries such as in the Untited States of America and New Zealand had a ratio of males to females getting the vaccination closer to 1 , meanwhile countries like Panama and Uruguay had ratios of male to female vaccination of 0.6 and 0.2 respectively (35).

Barriers to vaccination in low-income countries. This section tackles barriers associated with primary prevention strategies such as: cost of vaccination and implementation of such programs in low-income countries, sex as a barrier to male vaccination uptake, distance and transportation related issues leading to lower uptake of vaccination and lack of knowledge and stigma related to HPV vaccination in societies where this is still viewed as a taboo topic.

Financial cost of vaccination. The costs of vaccinations may also pose a major barrier to lowering the incidence of CC. In a study conducted among Chinese women, the high cost of the HPV vaccine served as one of the components of women's hesitancy to get vaccinated (36). This obstacle applies to several low HDI countries in which the individuals are expected to cover the cost of the vaccination. Studies have shown that governmentally sponsored programmes can be cost effective. In a study conducted in Thailand it was shown that if a catch-up program was implemented to vaccinate young women who were not previously vaccinated, it would cost less than 10,000 Thai Baht per Quality-of-Life Points (QUALYs). The recommended threshold for Thailand according to its government stood at 160,000 Thai Baht per QUALY proving that the implementation of a vaccination program would be cost effective (37). Therefore, it is important to consider the ultimate benefits of developing vaccination programmes for the health outcomes of the general population and the cost-effectiveness of such programmes. 
Sex and HPV vaccinations. HPV vaccination was initially introduced in the Untited States of America in 2006 and was approved as a 'females only' vaccine since its aim was the prevention of $\mathrm{CC}$. Following the initial introduction of the vaccine to women, it was soon realised that the latter did not stop men from infecting women with the virus and vice versa, and it became clear that it was pertinent to have both sexes protected. The initial notion was that if all women were vaccinated the latter could provide herd immunity for men, but this assumption was false (due to incomplete vaccination in all females) as well as the fact that homosexual men would still be vulnerable to infection with HPV (38). Therefore, in the year 2009, HPV vaccination was approved as a sex-neutral vaccination. The current guidelines by the Advisory Committee on Immunization Practices recommend vaccination of young girls and boys at age 9-14 years old with the two-dose option (39).

While low rates of HPV vaccination generally may be a problem of more rural communities, the feminisation of HPV persists in high HDI countries. According to a study in the Untited States of America in 2014 approximately $60 \%$ of females aged 13 to 17 received at least one dose of the vaccine compared to only $42 \%$ of their male counterparts. The catch-up programme in 2012 showed even lower levels of vaccination with $34 \%$ of women and $5.5 \%$ of men aged 18 to 26 respectively (38).

School based vaccinations should be considered in an effort to increase vaccination rates among young people. By following a school-based vaccination programme, Australia was able to reach a vaccination coverage of $86 \%$ of females and $78 \%$ of males respectively. This success could also be attributed to strong community acceptance for the vaccine as a tool for cancer prevention in both sexes (40).

Distance and transportation. Another point of concern for low HDI country parents was having to take their child out of school for multiple doses of the vaccine, especially taking into consideration the transport limitations addressed above (41).

Lack of knowledge and stigma. Studies have reported that parents and spouses of women participating in prevention programs in resource-depleted settings were concerned regarding HPV vaccination since in their opinion this would affect the fertility of the women. Some also believed that HPV vaccination had association with the human immunodeficiency virus (HIV), which is also stigmatised and hence served as a barrier to screening. Some parents also believed that intimate examinations and HPV vaccination in their daughters would initiate early coitarche and this led to reluctancy in medical follow-up. In these communities, diagnoses of CC may also be viewed as a death sentence despite advancements in treatment, so some women opt out of prevention programs under the belief that they would rather die not knowing or that it is a religion-guided fate (41). Another misconception conveyed is that only women need to get the vaccine as this is purely a biologically female problem (42).

\section{Psychoeducation}

Psychoeducation of the general population about $\mathrm{CC}$ and its associated risk factors is a form of primary prevention that is not given enough attention. To highlight this, a study in Kenya was performed in which one third of the interviewed women aged 15-24 did not know what $\mathrm{CC}$ even is (30). Lack of education and poverty are greatly associated with this knowledge gap, but even high HDI countries have individuals with limited understanding on how to protect themselves from CC. Interestingly, according to a study in the United Kingdom, information leaflets increase knowledge on CC symptomatology, lower overall anxiety and prompt help-seeking (43). Nevertheless, some individuals may find CC related information too challenging and hence feel discouraged to partake in HPV vaccination or screening. For example, another United Kingdom based study aimed to identify the challenges associated with the National Health Service leaflet. Some of the main issues encountered included not understanding the benefits or results of screening, the risks of screening and HPV infection, or complex terminology (44). Other leaflets in Scandinavian countries such as Norway, Finland were reported to not address the HPV related information that was truly sought after by the audience, such as psychosocial information or unbiased alternatives to vaccination and screening (45).

$\mathrm{Ngu}$ et al (46) conducted a study in which participants were allocated into groups. The firs group was offered counselling along with a leaflet, whereas the second group was only provided with a leaflet. The study then compared CC related anxiety, depression and screening related knowledge levels in the two groups of women. In this study, the two groups were followed up with a questionnaire pre-intervention, a week after and 6 months after the intervention. The researchers reported that the counselling group had a higher score in knowledge on screening and HPV at 1 week follow-up; yet at a 6-month follow-up both the intervention and control groups had approximately the same level of knowledge on CC screening and risks. Meanwhile $\mathrm{CC}$ related anxiety gradually decreased in both groups equally (46). This trial demonstrates that it is not mandatory to implement costly information services such as personal counselling, and that even leaflets with basic guidelines could be impactful in low HDI regions. Very limited information can be found on the effects of psychoeducational programs and leaflets on populations in resource depleted settings, which implies that psychoeducation programmes are not common in low income countries. Of course, such programs may not be the sufficient to achieve CC elimination, but they may be beneficial. Therefore, educational interventions from a young age could be a powerful tool for prevention of $\mathrm{CC}$ incidence.

Barriers to psychoeducation. The cost of educational programme can be a significant obstacle to lowering the burden of CC. A randomised cluster trial among uninsured Hispanic women examined the benefits of having a group-based educational session which is also culturally appropriate by a professional in the field. Additionally, the study also provided free of charge screening for all the women in the study. While the benefits of these interventions were clear, the funding was provided by the Institute conducting the research (47). Hence, the cost of such interventions would be something inaccessible for these women in their normal setting. Another study in Peru highlighted that while in person educational interventions may be costly, an online alternative could have been a 
solution. However, some young women in low HDI countries do not even have access to internet, let alone information on CC primary prevention (48).

\section{Secondary prevention and challenges faced by developing countries}

Secondary prevention emphasizes early disease detection and its target is healthy-appearing individuals with subclinical forms of the disease. Secondary prevention screens to identify preneoplastic high grade lesions such as CIN II and III as well as identify $\mathrm{CC}$ in its earliest form, preferably before the onset of symptoms and signs, with screening being the most widely established form of secondary prevention in the context of CC (29). It is crucial for screening to cover a high coverage of the target population, specifically in the age groups of 25 to 65 years of age. Secondary prevention should also involve compliance with repeating screening tests according to the $\mathrm{CC}$ prevention program of the country as well as monitoring the results for comparison in order to see if there is evolution of any possible lesions and following up carefully screen-positive women.

Screening methods for $\mathrm{CC}$ range from conventional methods that have been available for many years, such as cytology, HPV testing and visual inspection with acetic acid (VIA), to more recent advancements such as self-sample kits and screening for methylation markers which are still undergoing research. Cytology is the most conventional method of secondary prevention due to its high uptake in several countries, while HPV testing for DNA sequences of the 12/14 high-risk HPV types is still under implementation in many countries (49). Self-sample kits constitute a different way of collecting cervico-vaginal cells (suitable for HPV testing and not for cytology) and represents a more comfortable method to increase women's participation to screening especially among stigmatised populations (50). While methylation markers are under study and currently used for triage of screen-positive women and are not as a primary screening tool. Since they are expensive and require well equipped laboratories, they are less suitable for middle-low income countries; yet more research in this field could be beneficial for CC patients. Different methods of screening therefore entail varying costs, and different requirements for infrastructure as well and expertise of personnel, which may be important obstacles in the implementation of successful screening programs in developing countries (49).

There is variation in screening uptake in Europe, with some countries having a fully implemented nation-wide screening program such as the Netherlands and Turkey, while France is still implementing a screening program that would be accessible to hard-to-reach populations, reduce CC incidence and deaths by $30 \%$, and reach an $80 \%$ screened population coverage over the next 10 years from $2019(51,52)$. Belgium and Switzerland have had a mean stable screening coverage of 70.9 and $73.1 \%$ during 2008-2012, respectively (53).

In the Untited States of America there was a decline in in overall screening in all age groups from 2010-2014. For example, women aged 30-39 had a decrease in screening uptake from 88 to $77.5 \%$ over the 4-year period, yet rates of co-testing were the highest compared to all other age groups standing at 44.4\% in 2014 (54). The significance of screening is supported by a population-based control trial in New Mexico which highlighted that there were 83 and $48 \%$ lower odds ratio of developing non-localised CC and localised CC for women screened 3 years prior to detection compared to women who did not undergo screening (55). Another retrospective study on a population also in New Mexico showed that out of 504 women diagnosed between 2009-2016, only $36 \%$ were screened at some point in their life. This result again reinforces the point of early detection and treatment as key for preventing debilitating complications and indeed this study confirmed that screening could have prevented $35 \%$ of these cases (56).

A cluster randomised trial in India with over 130,000 women also highlighted the benefit and statistical significance of screening for $\mathrm{CC}$. There were 34 deaths from $\mathrm{CC}$ in the HPV testing group vs. 64 deaths in the control group, hazard ratio $0.52 ; 95 \%$ confidence interval $0.33-0.83$ (57). Bhutan on the other hand was one of the first low to middle income countries to introduce a national HPV vaccination program in 2010, which also became sex neutral in 2020. However, despite being free of charge, screening uptake was only $59.2 \%$ among woman aged 18-69 years old and only increased to $66 \%$ in 2017 , supporting the need for greater efforts to be implemented to achieve a higher vaccination rate (58).

Apart from lowering disease burden and mortality, screening methods are economically more efficient than detecting the cancer at later stages and resorting to more costly, technical treatments. In fact, in the Untited States of America, it was estimated that a perfect compliance to screening at 3-year intervals would increase the incremental net monetary benefits from $\$ 759$ to $\$ 1,645$ as well as gain cheaper QUALYs (59).

\section{Recent developments in screening methods}

Cytology. Cytology has been a conventional method of screening since the 1940s, with many countries such as the UK and US developing screening programs based around this test. Unfortunately, though, this screening method has plenty of drawbacks such as high false negative rates due to sampling and interpretation errors, as well as artefacts impeding visualisation of the samples. This led to the emergence of alternative cytology-based techniques such as liquid based cytology, which holds numerous advantages over the conventional method. It has been shown to be faster, more efficient, more accurate, with fewer artefacts that could obscure the results as well as an overall higher detection rate of $97.60 \%$ as opposed to conventional cytology with a $71.96 \%$ detection rate respectively (60). It has been estimated that the liquid-based preparations have a sensitivity of $61-66 \%$ and a specificity of $82-90 \%$ as opposed to the conventional cytology preparation which has sensitivity of $51 \%$ and specificity of $57 \%$ (61). It has also been suggested that double reading of cytology slides could be implemented to avoid missing out on potential high grade squamous intraepithelial lesions (HSIL+). A study conducted in Mexico reported that 3,914 women with positive high-risk HPV were triaged with liquid-based cytology. The study detected a $20.9 \%$ increase in HSIL+ cases when double reading was done (62). 
Visualisation with acetic acid or Lugol's iodine. Another widely applied form of screening is visualisation with either acetic acid or Lugol's iodine, which is utilised extensively even today in marginalised populations. The technique has numerous benefits such as low cost, safety, immediate real time results permitting a single visit, as well as the possibility for a screen and treat approach without the fear of losing patients to follow-up (63). A further advantage of this technique is that it can be performed by healthcare personnel such as nurses and midwives after short training programs, without the need for a doctor to be present for the procedure to be implemented. This is especially crucial in resource depleted countries. The most remarkable limitation of this technique lies on its sensitivity and specificity, which were reported to be only 69.6 and $51.0 \%$ respectively for lesions of CIN II and above in a study carried out in Kenya (64). These rates are lower than the sensitivity and specificity of traditional liquid-based cytology. Another drawback may be that the healthcare personnel performing the technique may not be experienced enough to distinguish VIA positive from VIA negative patients. Recently in a study by Kudva et al (65) an Android Device based screening method was proposed to address this issue. The novel technique suggests that during VIA examination the health care provider uses a speculum with an Android device attached to acquire images of the cervix prior to and after acetic acid application, as well as the patient's identification details. Upon acquisition, these images are sent to a medical expert in the field, who after examination deems them VIA positive or negative. In a clinical study involving more than 100 women in India, it was found that the algorithm offered a sensitivity of $99.05 \%$ and a specificity of $97.16 \%(65)$.

HPV DNA genome assays. Testing for high-risk HPV genomes is a relatively reliable option for detection of CIN. Despite studies demonstrating that HPV testing has a lower specificity $(89.9 \%)$ than cytology $(96.3 \%)$ for CINII, this screening method still has a number of advantages (66). However, in accordance with the most recent guidelines, primary HPV testing must be performed by assays targeting all the high-risk types (HPV 16, 18, 31, 33, 35, 39, 45, 51, 52 , $56,58,68)$, that have been clinically validated according to the international recommendations published in 2009 and in a recent study by Arbyn et al (67), a list of 11 DNA assays fulfilled all requirements in order to serve as a primary screening tool.

Ronco et al (68) conducted a pooled analysis of 4 major European trials comparing HPV testing to cytology. The results of this study showed that where cumulative incidence was investigated, HPV screening was superior to cytology at 3.5 and 5.5 year follow-up. The experimental group (HPV screening) and control group (cytology screening) had to have negative tests at the starting point. At the 2 follow-ups the experimental group had a cumulative incidence of $\mathrm{CC}$ of 4.6 and 8.7 per 100,000 women while the cumulative incidence of the control group was 15.4 and 36.0 per 100,000 women respectively. Therefore, there was a discrepancy of approximately $70 \%$ across the two methods (68). It should be noted that HPV screening offers the option of self-sampling for HPV, thereby eliminating the need for a health care provider and the time consuming and possibly anxiety-inducing waiting period for the patient's appointment. This could be particularly relevant for women from backgrounds in which such procedures are highly stigmatised.

An example of testing for HPV DNA includes the careHPV testing system (Qiagen), which can evaluate 90 samples from patients simultaneously. It is a fast and portable system designed specifically for low-income countries. A prospective cohort study investigated whether self-sampling specimens were of lower quality than physician obtained specimens. However, the analysis of the results showed that there was little difference in sensitivity between the two groups; yet the comfort level of the self-sampling group was significantly higher (69). Another study held in Vancouver also aimed to test the feasibility of using self-assessment kits in women for detecting HPV DNA. Women from shelters and women centres were recruited and $28.5 \%$ of these women tested positive for the virus. Approximately $14 \%$ of the participants never had a cytological analysis before compared to the local general population in which $8.3 \%$ never carried out cytology (70). Hence this screening method should be considered for implementation in certain geographical regions with higher stigma surrounding collecting cervical swabs.

Recently efforts have been made to develop tests to investigate the presence of HPV in the urine. A study in the UK compared the presence of HPV in urine vs cervical samples. The results of the study showed that the detection of HPV in the urine was comparable to the detection of the virus in cervical samples; yet urine samples had lower sensitivity. Despite the lower sensitivity, developing methods that detect HPV in urine may prove useful for women not attending cervical screening (71).

One noteworthy drawback in HPV testing alone as a primary screening test is the proportion of women with a positive result due to only a transient infection with a viral HPV strain, which however may not be clinically relevant. To exemplify this, in a study of over 30,000 screened women $11 \%$ were initially shown to be HPV positive but upon subsequent re-testing the prevalence of high-risk HPV was 1.5\% (72). Part of the decrease could be attributed to the fact that general HPV screening is not specific enough and will identify all types of HPV strains instead of only the high-risk strains.

Based on the above-mentioned data, one could suggest that combining 2 high sensitivity and specificity screening methods would yield better prospects. In a study conducted in Mexico 3 screening groups (liquid-based cytology, HPV genotype 16/18 screening and both combined) were compared and the respective sensitivity and specificity for each of the 3 groups were 42.9 and $74 \%, 58.3$ and $54.4 \%, 86.6$ and $34 \%$. The referral rate for colposcopy as the next step was $29 \%$ for the group with both screening methods as compared to liquid-based cytology alone which was $12 \%$ (73). Meanwhile another study in New Mexico has shown that in women aged 30-64 years with negative cytology, the proportion of screening tests that were co-tests (i.e., using two screening methods instead of one) rose from $5.6 \%$ in 2008 to $84.3 \%$ in 2019. However, despite the increase in co-tests, it has been demonstrated to be unnecessary in primary screening, since it does not increase sensitivity and does not affect specificity while at the same time it increases the financial cost and 
may cause a major financial impact especially for low HDI countries (74).

A promising advancement in tertiary prevention is the combination of HPV screening along with p16INK4a immunostaining which is a promising biomarker for the triage of HPV positive women. Analysis from trials suggests that using these 2 methods simultaneously vs. using cytology alone results in a $53 \%$ higher detection rate of CINII-CINIII (75). However, this is still a novel screening suggestion and warrants further trials. p16INK4a immunohistochemistry also plays a role on classifying CINII as either high grade or low grade, which can be particularly relevant for women under 30 years old since low grade CINII in this age group commonly relies on surveillance as opposed to more radical interventions. The p16 immunohistochemistry positivity was associated with higher severity CIN and higher risk HPV strains with P-value being below 0.001 in studies, which is beneficial information in terms of patient management (76). A particularly interesting aspect of p16 immunohistochemistry in another study was that it was positive in CC in cases where HPV was negative. This indicates that p16 immunohistochemistry could be possibly used as a screening marker when other more conventional tests fail (69). However, there are only a few RCTs to assess the efficacy of implementation of these particular sequences of tests, which WHO acknowledges as a field for further investigation.

HPV RNA assays. Research is currently conducted to investigate whether HPV RNA assays can be used as an adjunctive method for cervical cancer screening. A study has shown that the specificity for HPV RNA assay was $96.3 \%$ compared to the specificity of ordinary HPV DNA assay which was $94.8 \%$. These results support the use of RNA assays as an effective concurrent CC screening method to be used alongside other more conventional screening methods that have high sensitivity but lower specificity (70). Another study evaluated the accuracy of the mRNA HPV biomarker in cervical smears to identify cervical intraepithelial neoplasia (CIN) 2 or 3 and cervical cancer. The results showed that the HPV mRNA test exhibited overall percentage agreement with histological diagnosis of $89.8 \%$. It was concluded that the HPV mRNA assays may be an adequate tool in the secondary screening of cervical cancer (77).

Methylation biomarkers. A new field of investigation lies in the emergence of methylation markers as a screening method for CC. Over the last few years, it has been noted that alterations in host and viral DNA methylation levels occur during the development of the carcinoma, so establishing the specific changes in the DNA methylation involved could provide insight on early detection and treatment (78).

A study among Slovenian HPV positive women was carried out to screen for certain clusters of methylation markers and yielded some promising results. Methylation markers C13orf18, EPB41L3, JAM3 had $80 \%$ sensitivity and $66 \%$ specificity for CINII and greater, while the SOX1 and ZSCAN1 combination demonstrated $63 \%$ sensitivity and $84 \%$ specificity for the same CIN type. Furthermore, the markers for both groups had higher sensitivity as the neoplasia progressed from CINII to CINIII (79). Another study investigating the methylation status of PAX1, ZNF582, SOX1, and NKX6-1 found that dual methylation of PAX1 and ZNF582 showed a 100\% association with cervical pathological processes. The sensitivity and specificity for these markers in identification of CINIII were 78.85 and $73.55 \%$ respectively (80). It is worth noting that methylation marker studies aside from being very specific and sensitive, they can accurately detect CIN3 and malignancy irrespective of geographic context and setting (81).

In a blinded case-control study, methylation markers as a screening tool were compared to HPV with cytology screening based on sensitivity and specificity. The results demonstrated that the sensitivity and specificity for CINIII methylation markers were 93.2 and $41.8 \%$ as opposed to combined HPV and cytology which were 86.4 and $49.8 \%$ respectively (82). These results propose that methylation markers may be more sensitive than conventional CC screening tests. In another study, the methylation status of selected sites on HPV16 and human genes in DNA were examined. The results showed that methylation of viral HPV16L1 and host sites, EPB4L3 and LMX1, strongly discriminates CIN2/3 from NILM/CIN1 and cancer from CIN2/3 and may therefore be used as both a diagnostic as well as a prognostic marker (83). Despite the promising results, plenty of work persists in identifying the most sensitive cluster of methylation markers that would be predictive of $\mathrm{CC}$ at earlier stages.

\section{Barriers to screening in low resource countries and strategies for improvement}

European guidelines offer ample information for screening strategies at country level such as which screening tests to utilise, age range for screening, management upon positive tests, but it is up to each individual country to integrate these costly recommendations. Upon diagnosis of CC there are two clear groups identified: women of higher socio-economic status who test positive early and women with a vulnerable socio-economical profile who often test positive further in the course of their disease. In European countries this disparity is addressed via individual invite of the target population to screening based on a central nation registry, which insures equal access to the screening programme. Low income countries face higher difficulty due to financial limitations and higher burden of $\mathrm{CC}$ and still have room for improvement. Adequate targeting of infrastructure, geographical inaccessibility, structured time-management in clinics, patient education and effective communication between provider and patient, combination of vaccination and screening, analysing and improving screening programmes could offer considerable improvement in screening in vulnerable countries (84).

Lack of infrastructure and financial resources. Lack of infrastructure and limited financing may pose a challenge for screening. In a study comparing resources for screening in hospitals in Eastern, Southern and Central Africa, it was found that some financial limitations persist in these areas. In some Tanzanian hospitals for example, there was a shortage of fixating agents for cytology. Other hospitals also reported lack of cytology technicians. Out of the 12 African hospitals examined only 2 offered cryotherapy and loop excision, meanwhile all the others could only offer core biopsy (85). Another study in rural Kenya provided evidence that lack of staff, inadequate 


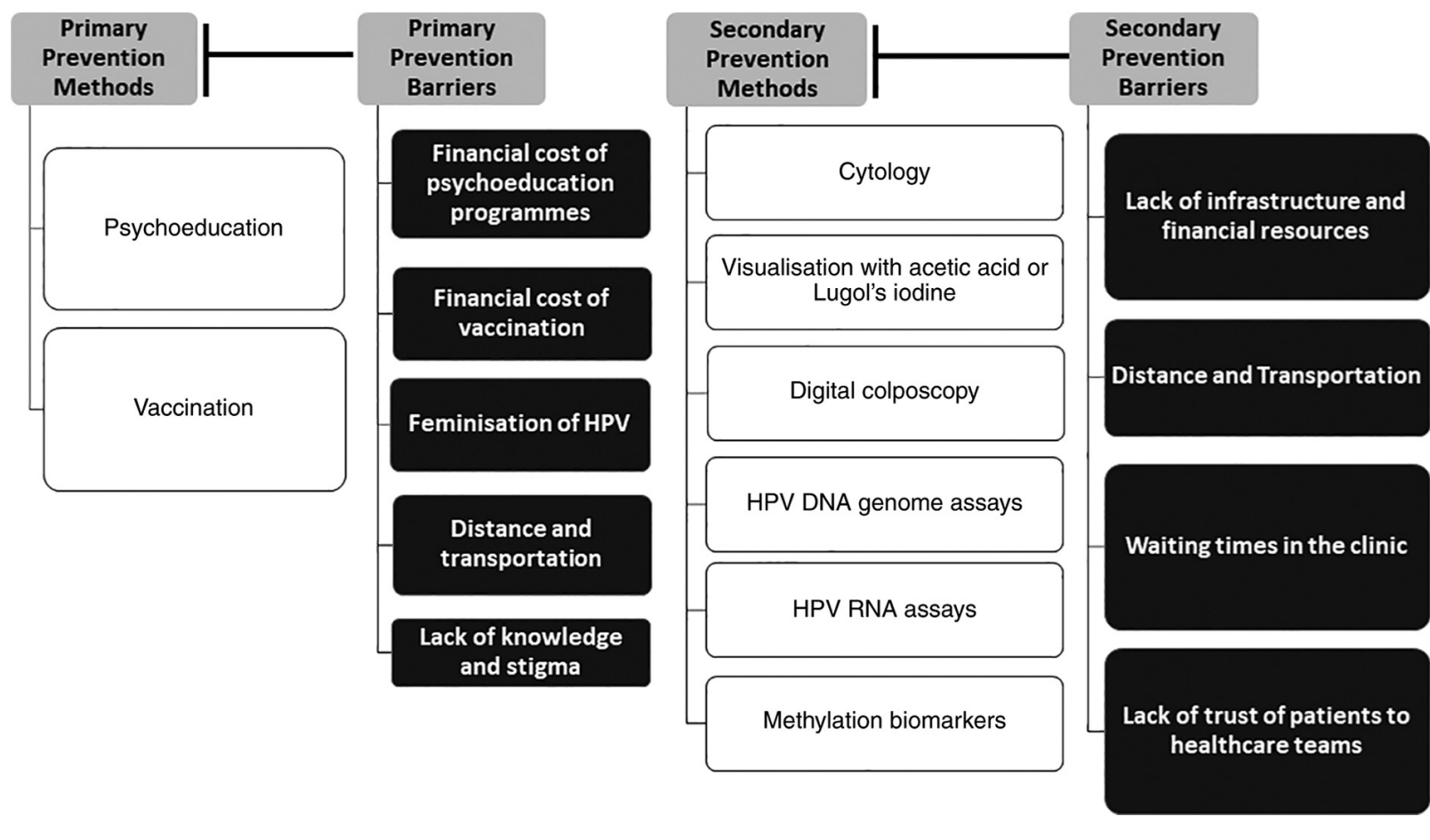

Figure 1. Flow chart on primary and secondary prevention methods, as well as common barriers to prevention. HPV, human papilloma virus.

training, limited space, lack of supplies for screening lack of an autoclave were common challenges. Moreover, the majority of staff members desired more training in the field (86).

Geographic accessibility burden. In some low HDI countries, reaching a facility with a screening programme may be a considerable obstacle. A study in Mexico showcased that woman who resided in rural areas had a significantly greater geographic accessibility burden when compared to nonrural areas (4.4 vs. $2.5 \mathrm{~km}$ for screening; 9.9 vs. $4.2 \mathrm{~km}$ for colposcopy; and 14.8 vs. $6.6 \mathrm{~km}$ for precancer treatment services, all $\mathrm{P}<0.001)$.

Furthermore, women in rural areas had to cover an extra 5.7 and $8.2 \mathrm{~km}$ for access to colposcopy and precancer treatment services respectively (87). Another study in Kenya showcased that some women needed up to $8 \mathrm{~h}$ to travel to the nearest screening site as well as rely on taxi services. The cost of the taxi itself would be equal to the wages a woman would make in agricultural work (88). Therefore, lack of transportation should also be considered as a barrier.

Waiting times in the clinic. The waiting time in rural clinics may also be an obstacle to screening. For example, in a study in Serbia it was shown that although medical personnel were available, waiting time for screening could take up to $4 \mathrm{~h}$. Apart from having to wait in overcrowded waiting areas, women felt that the providers did not answer their queries or show the anticipated respect to the patients, which also contributed to women not wanting to attend follow-up appointments (89).

Lack of trust of patients to healthcare teams. The study by Urasa and Darj (90) conducted in Tanzania addressed the lack of knowledge of nurses regarding the aetiology of CC and how the latter hindered the trust of the patients. Lack of knowledge from the medical personnel made the women more reluctant to undergo screening, especially since this is viewed as a taboo topic in the community (90). The study emphasised the importance of training and continuous professional development of the healthcare team involved in CC screening.

\section{Conclusion}

Despite CC contributing so greatly to the overall cancer mortality rate, it is also one of the most well researched cancers, with a plethora of prevention methods. It is clear that there is a disparity in primary and secondary prevention programmes in high vs. low HDI countries. Despite the development of effective methods of primary and secondary prevention, numerous obstacles are faced which should be addressed to ensure the prevention programmes are effective (Fig. 1). Lack of psychoeducation, access to vaccination, stigma but mostly the lack of implementation of a stable and accessible screening program in low HDI countries due to the lack of infrastructure and financial costs involved is the epicentre of the problem. Research has focused on addressing the needs of developing countries by trying to develop novel and economically more cost-effective and accessible screening methods. The effectiveness of vaccines for $\mathrm{CC}$ as well as the multiple screening options related to it, should call for immediate enforcement of well-structured and easily accessible primary and secondary prevention programmes by policy makers, especially in more resource depleted countries which currently have the highest incidence of invasive pathology. Introduction of such prevention programmes across all geographical regions and maintenance of high adherence rates by women via continuous psychoeducation would lead to $\mathrm{CC}$ becoming a worry of the past. 


\section{Acknowledgements}

Not applicable.

\section{Funding}

No funding was received.

\section{Availability of data and materials}

Not applicable.

\section{Authors' contributions}

$\mathrm{AB}, \mathrm{CA}$ and $\mathrm{CC}$ were all involved in the conceptualization of the current manuscript. $\mathrm{AB}$ and $\mathrm{CC}$ were involved in the literature search and review of the resources that were used in the current manuscript. $\mathrm{AB}, \mathrm{CA}$ and $\mathrm{CC}$ were involved in the writing and revision of the manuscript. All authors read and approved the final manuscript. Data authentication is not applicable.

\section{Ethics approval and consent to participate}

Not applicable.

\section{Patient consent for publication}

Not applicable.

\section{Authors' information}

DrConstantina Constantinou: Orcid ID:0000-0001-6167-4023.

\section{Competing interests}

The authors declare that they have no competing interests.

\section{References}

1. Globocan 2020: New Global Cancer Data. In: Uicc.org. 2021 https://www.uicc.org/news/globocan-2020-new-global-cancer-data. Accessed September 18, 2021

2. Al Jishi T and Sergi C: Current perspective of diethylstilbestrol (DES) exposure in mothers and offspring. Reprod Toxicol 71: 71-77, 2017.

3. Adcock R, Cuzick J, Hunt WC, McDonald RM and Wheeler CM; New Mexico HPV Pap Registry Steering Committee: Role of HPV genotype, multiple infections, and viral load on the risk of high-grade cervical Neoplasia. Cancer Epidemiol Biomarkers Prev 28: 1816-1824, 2019.

4. Doorbar J, Egawa N, Griffin H, Kranjec C and Murakami I: Human papillomavirus molecular biology and disease association. Rev Med Virol 25 (Suppl): S2-S23, 2015.

5. Galloway D and Laimins L: Human papillomaviruses: Shared and distinct pathways for pathogenesis. Cur Opin Virol 14: 87-92, 2015.

6. Plummer M, de Martel C, Vignat J, Ferlay J, Bray F and Franceschi S: Global burden of cancers attributable to infections in 2012: A synthetic analysis. Lancet Glob Health 4: e609-e616, 2016.

7. Wagner M, Bennetts L, Patel H, Welner S, de Sanjose S and Weiss T: Global availability of data on HPV genotype-distribution in cervical, vulvar and vaginal disease and genotype-specific prevalence and incidence of HPV infection in females. Infect Agent Cancer 10: 13, 2015.
8. Yuan Y, Cai X, Shen F and Ma F: HPV post-infection microenvironment and cervical cancer. Cancer Lett 497: 243-254, 2021.

9. Cohen O, Schejter E, Agizim R, Schonman R, Chodick G, Fishman A and Hershko Klement A: Postcoital bleeding is a predictor for cervical dysplasia. PLoS One 14: e0217396, 2019.

10. Reed N, Balega J, Barwick T, Buckley L, Burton K, Eminowicz G, Forrest J, Ganesan R, Harrand R, Holland C, et al: British Gynaecological Cancer Society (BGCS) cervical cancer guidelines: Recommendations for practice. Eur J Obstet Gynecol Reprod Biol 256: 433-465, 2021.

11. Issa Z, Aloan $\mathrm{H}$ and Al Niyazee A: Accuracy of Pap smear in cervical cancer screening. World Family Med J 17: 16-20, 2019.

12. Johnson CA, James D, Marzan A and Armaos M: Cervical cancer: An overview of pathophysiology and management. Semin Oncol Nurs 35: 166-174, 2019.

13. Pkhakadze G, Bokhua Z, Asatiani T, Muzashvili T and Burkadze G: Evaluation of the risk of cervical intraepithelial neoplasia progression based on cell proliferation index, epithelial-mesenchymal transition and co-infections. Georgian Med News: 178-184, 2020.

14. Bhatla N, Aoki D, Sharma DN and Sankaranarayanan R: Cancer of the cervix Uteri. Int J Gynaecol Obstet 143: 22-36, 2018.

15. Li X, Xia L, Chen X, Fu Y and Wu X: Simple conization and pelvic lymphadenectomy in early-stage cervical cancer: A retrospective analysis and review of the literature. Gynecol Oncol 158: 231-235, 2020.

16. Landoni F, Maneo A, Zapardiel I, Zanagnolo V and Mangioni C: Class I versus class III radical hysterectomy in stage IB1-IIA cervical cancer. A prospective randomized study. Eur J Surg Oncol 38: 203-209, 2012.

17. Gopalani SV, Janitz AE and Campbell JE: Cervical cancer incidence and mortality among Non-Hispanic African American and white women, United States, 1999-2015. J Natl Med Assoc 112: 632-638, 2020

18. Lott BE, Trejo MJ, Baum C, McClelland DJ, Adsul P, Madhivanan P, Carvajal S, Ernst K and Ehiri J: Interventions to increase uptake of cervical screening in Sub-Saharan Africa: A scoping review using the Integrated Behavioral Model. BMC Public Health 20: 654, 2020.

19. International agency for research on cancer. Cervix uteri fact sheet. 2020. https://gco.iarc.fr/today/data/factsheets/cancers/23Cervix-uteri-fact-sheet.pdf. Accessed October 28, 2021.

20. AylwardJGlobalburden of cervicalcancer.In:TogetHER forHealth. 2021. https://togetherforhealth.org/global-burden-cervical-cancer/. Accessed November 28, 2021.

21. Keenan M: Human Development Index (HDI). In: Salem Press Encyclopedia. 2021. https://search.ebscohost.com/login.aspx?dir ect=true $\&$ AuthType $=i p, s s o \& d b=e r s \& A N=98402115 \&$ site $=$ edslive. Accessed September 28, 2021.

22. Arbyn M, Weiderpass E, Bruni L, de Sanjosé S, Saraiya M, Ferlay $\mathrm{J}$ and Bray $\mathrm{F}$ : Estimates of incidence and mortality of cervical cancer in 2018: A worldwide analysis. Lancet Glob Health 8: e191-e203, 2020.

23. Burton-Jeangros C, Cullati S, Manor O, Courvoisier DS, Bouchardy C and Guessous I: Cervical cancer screening in Switzerland: Cross-sectional trends (1992-2012) in Social Inequalities. Eur J Public Health 27: 167-173, 2017.

24. Lam JO, Lim WY, Chow KY and D'Souza G: Incidence, trends and ethnic differences of oropharyngeal, anal and cervical cancers: Singapore, 1968-2012. PLoS One 10: e0146185, 2015.

25. Garau M, Musetti C, Alonso R and Barrios E: Trends in cancer incidence in Uruguay: 2002-2015. Colomb Med (Cali) 50: 224-238, 2019.

26. Gopalani S, Janitz A and Campbell J: Cervical Cancer Incidence and Mortality among Non-Hispanic African American and White Women, United States, 1999-2015. J Natl Med Assoc 112: 632-638, 2020.

27. Lott B, Trejo M, Baum C, McClelland D, Adsul P, Madhivanan P, Carvajal S, Ernst K and Ehiri J: Interventions to increase uptake of cervical screening in sub-Saharan Africa: A scoping review using the integrated behavioral model. BMC Public Health 20: $654,2020$.

28. Lekoane K, Kuupiel D, Mashamba-Thompson T and Ginindza T: Evidence on the prevalence, incidence, mortality and trends of human papilloma virus-associated cancers in Sub-Saharan Africa: Systematic scoping review. BMC Cancer 19: 563, 2019.

29. Kisling LA: Prevention strategies. In: StatPearls. 2021. https://www.ncbi.nlm.nih.gov/books/NBK537222/. Accessed September 15, 2021. 
30. Von Karsa L, Arbyn M, De Vuyst H, Dillner J, Dillner L, Franceschi S, Patnick J, Ronco G, Segnan N, Suonio E, et al: European guidelines for quality assurance in cervical cancer screening. Summary of the supplements on HPV screening and vaccination. Papillomavirus Res 1: 22-31, 2015.

31. Banday A, Jeelani S and Hruby V: Cancer vaccine adjuvants-recent clinical progress and future perspectives. Immunopharmacol Immunotoxicol 37: 1-11, 2014.

32. Bogani G, Leone Roberti Maggiore U, Signorelli M, Martinelli F, Ditto A, Sabatucci I, Mosca L, Lorusso D and Raspagliesi F: The role of human papillomavirus vaccines in cervical cancer: Prevention and treatment. Crit Rev Oncol Hematol 122: 92-97, 2018.

33. Bosch F, Robles C, Díaz M, Arbyn M, Baussano I, Clavel C, Ronco G, Dillner J, Lehtinen M, Petry K, et al: HPV-FASTER: Broadening the scope for prevention of HPV-related cancer. Nat Rev Clin Oncol 13: 119-132, 2016.

34. Cuzick J and Wheeler C: Need for expanded HPV genotyping for cervical screening. Papillomavirus Res 2: 112-115, 2016.

35. Bruni L, Saura-Lázaro A, Montoliu A, Brotons M, Alemany L, Diallo MS, Afsar OZ, Lamontagne DS, Mosina L, Contreras M, et al: HPV vaccination introduction worldwide and WHO and UNICEF estimates of national HPV immunization coverage 2010-2019. Prev Med 144: 106399, 2021.

36. Siu J, Fung T and Leung L: Social and cultural construction processes involved in HPV vaccine hesitancy among Chinese women: A qualitative study. Int J Equity Health 18: 147, 2019.

37. Termrungruanglert $\mathrm{W}$, Khemapech $\mathrm{N}$, Vasuratna A, Havanond $\mathrm{P}$, Deebukkham P, Kulkarni A and Pavelyev A: The epidemiologic and economic impact of a quadrivalent human papillomavirus vaccine in Thailand. PLoS One 16: e0245894, 2021.

38. Daley E, Vamos C, Zimet G, Rosberger Z, Thompson E and Merrell L: The Feminization of HPV: Reversing gender biases in US Human papillomavirus vaccine policy. Am J Public Health 106: 983-984, 2016.

39. Daley E, Vamos C, Thompson E, Zimet G, Rosberger Z, Merrell L and Kline N: The feminization of HPV: How science, politics, economics and gender norms shaped U.S. HPV vaccine implementation. Papillomavirus Res 3: 142-148, 2017.

40. Patel C, Brotherton J, Pillsbury A, Jayasinghe S, Donovan B, Macartney $\mathrm{K}$ and Marshall $\mathrm{H}$ : The impact of 10 years of human papillomavirus (HPV) vaccination in Australia: What additional disease burden will a nonavalent vaccine prevent? Euro Surveill 23: 1700737, 2018.

41. Chidyaonga-Maseko F, Chirwa M and Muula A: Underutilization of cervical cancer prevention services in low and middle income countries: A review of contributing factors. Pan Afr Med J 21 $231,2015$.

42. Jaiswal J, LoSchiavo C, Maiolatesi A, Kapadia F and Halkitis P: Misinformation, Gendered perceptions, and low healthcare provider communication around HPV and the HPV vaccine among young sexual minority Men in New York City: The P18 cohort study. J Community Health 45: 702-711, 2020.

43. Morris M, Friedemann Smith C, Boxell E, Wardle J, Simon A and Waller J: Quantitative evaluation of an information leaflet to increase prompt help-seeking for gynaecological cancer symptoms. BMC Public Health 16: 374, 2016.

44. Okan Y, Petrova D, Smith S, Lesic V and Bruine de Bruin W: How do Women interpret the NHS information leaflet about cervical cancer screening? Med Decis Making 39: 738-754, 2019.

45. Kolthoff S, Hestbech M, Jørgensen K and Brodersen J: Do invitations for cervical screening provide sufficient information to enable informed choice? A cross-sectional study of invitations for publicly funded cervical screening. J R Soc Med 109 274-281, 2016

46. Ngu S, Wei N, Kwan T, Chu M, Tse K, Chan K and Ngan H: Impact of different educational interventions on psychosocial well-being of women with a positive high-risk human papillomavirus and normal cervical cytology: A randomised trial. J Psychosom Obstet Gynaecol 39: 146-155, 2017.

47. Calderón-Mora J, Byrd TL, Alomari A, Salaiz R, Dwivedi A, Mallawaarachchi I and Shokar N: Group versus individual culturally tailored and theory-based education to promote cervical cancer screening among the underserved Hispanics: A cluster randomized trial. Am J Health Promot 34: 15-24, 2019.

48. Clavé Llavall A, de Wildt G, Meza G, Tattsbridge J and Jones L: Nurses' and teachers' perceived barriers and facilitators to the uptake of the human papilloma virus (HPV) vaccination program in Iquitos, Peru: A qualitative study. PLoS One 16: e0255218, 2021.
49. Catarino R, Petignat P, Dongui G and Vassilakos P: Cervical cancer screening in developing countries at a crossroad: Emerging technologies and policy choices. World J Clin Oncol 6: 281-290, 2015

50. Tranberg M, Bech B, Blaakær J, Jensen J, Svanholm H and Andersen B: Preventing cervical cancer using HPV self-sampling: Direct mailing of test-kits increases screening participation more than timely opt-in procedures-a randomized controlled trial. BMC Cancer 18: 273, 2018

51. Maver P and Poljak M: Primary HPV-based cervical cancer screening in Europe: Implementation status, challenges, and future plans. Clin Microbiol Infect 26: 579-583, 2020.

52. Woronoff A, Molinié F and Trétarre B: Implementation of national cervical cancer screening program in France. Bull Cancer 106: 253-261, 2019.

53. De Prez V, Jolidon V, Willems B, Cullati S, Burton-Jeangros C and Bracke P: Cervical cancer (over)screening in Belgium and Switzerland: Trends and social inequalities. Eur J Public Health 30: 410-415, 2020

54. Watson M, Benard V and Flagg E: Assessment of trends in cervical cancer screening rates using healthcare claims data: United States, 2003-2014. Prev Med Rep 9: 124-130, 2018.

55. Landy R, Sasieni PD, Mathews C, Wiggins CL, Robertson M, McDonald YJ, Goldberg DW, Scarinci IC, Cuzick J and Wheeler CM; New Mexico HPV Pap Registry Steering Committee: Impact of screening on cervical cancer incidence: A population-based case-control study in the United States. Int J Cancer 147: 887-896, 2019.

56. Landy R, Mathews C, Robertson M, Wiggins CL, Mcdonald YJ, Goldberg DW, Scarinci IC, Cuzick J, Sasieni PD and Wheeler CM: A state-wide population-based evaluation of cervical cancers arising during opportunistic screening in the United States. Gynecol Oncol 159: 344-353, 2020.

57. Sankaranarayanan R, Nene B, Shastri S, Jayant K, Muwonge R, Budukh AM, Hingmire S, Malvi SG, Thorat R, Kothari A, et al: HPV screening for cervical cancer in rural India. N Engl J Med 360: 1385-1394, 2009.

58. Dorji T, Tshomo U, Gyamtsho S, Tamang S, Wangmo S and Pongpirul K: Gender-neutral HPV elimination, cervical cancer screening, and treatment: Experience from Bhutan. Int J Gynaecol Obstet: Apr 30, 2021 (Epubs ahead of print). doi: 10.1002/ijgo.13728.

59. Kim JJ, Campos NG, Sy S, Burger EA, Cuzick J, Castle PE, Hunt WC, Waxman A and Wheeler CM; New Mexico HPV Pap Registry Steering Committee: Inefficiencies and High-value improvements in U.S. Cervical cancer screening practice. Ann Internal Med 163: 589-597, 2015.

60. Singh U, Anjum, Qureshi S, Negi N, Singh N, Goel M and Srivastava K: Comparative study between liquid-based cytology \& conventional Pap smear for cytological follow up of treated patients of cancer cervix. Indian J Med Res 147: 263-267, 2018

61. Karimi-Zarchi M, Peighmbari F, Karimi N, Rohi M and Chiti Z: A Comparison of 3 ways of conventional pap smear, liquid-based cytology and colposcopy vs cervical biopsy for early diagnosis of premalignant lesions or cervical cancer in women with abnormal conventional pap test. Int J Biomed Sci 4: 205-210, 2013.

62. Ramírez-Palacios P, Chen A, Flores YN, Crespi CM, Lazcano-Ponce E, Alvarez-Escobedo D, Torres-Ibarra L, Rivera-Paredez B,León-Maldonado L, Hernández-López R, et al: Benefit of double-reading cytology smears as a triage strategy among high-risk human papillomavirus-positive women in Mexico. Cancer Cytopathol 128: 715-724, 2020.

63. Lee H, Kang Y and Ju W: Cervical cancer screening in developing countries: Using visual inspection methods. Clin J Oncol Nurs 20: 79-83, 2016.

64. Orang'o E, Wachira J, Asirwa F, Busakhala N, Naanyu V, Kisuya J, Otieno G, Keter A, Mwangi A and Inui T: Factors associated with uptake of visual inspection with acetic Acid (VIA) for cervical cancer screening in western Kenya. PLoS One 11: e0157217, 2016.

65. Kudva V, Prasad K and Guruvare S: Andriod device-based cervical cancer screening for resource-poor settings. J Digital Imaging 31: 646-654, 2018

66. Koliopoulos G, Nyaga V, Santesso N, Bryant A, Martin-Hirsch P, Mustafa R, Schünemann H, Paraskevaidis E and Arbyn M: Cytology versus HPV testing for cervical cancer screening in the general population. Cochrane Database Syst Rev 8: CD008587, 2017. 
67. Arbyn M, Simon M, Peeters E, Xu L, Meijer CJLM, Berkhof J, Cuschieri K, Bonde J, Ostrbenk Vanlencak A, Zhao F, et al: 2020 list of human papillomavirus assays suitable for primary cervical cancer screening. Clin Microbiol Infect 27: 1083-1095, 2021.

68. Ronco G, Dillner J, Elfström KM, Tunesi S, Snijders PJ, Arbyn M, Kitchener H, Segnan N, Gilham C, Giorgi-Rossi P, et al: Efficacy of HPV-based screening for prevention of invasive cervical cancer: Follow-up of four European randomised controlled trials. Lancet 383: 524-532, 2014

69. Castle PE, Pierz AJ, Adcock R, Aslam S, Basu P, Belinson J, Cuzick J, El-Zein M, Ferreccio C, Firnhaber C, et al: A pooled analysis to compare the clinical characteristics of human papillomavirus-positive and -negative cervical precancers. Cancer Prev Res 13: 829-840, 2020.

70. Reid JL, Wright TC, Stoler MH, Cuzick J, Castle PE, Dockter J, Getman D and Giachetti C: Human papillomavirus oncogenic mRNA testing for cervical cancer screening. Am J Clin Pathol 144: 473-483, 2015.

71. Cuzick J, Cadman L, Ahmad AS, Ho L, Terry G, Kleeman M, Lyons D, Austin J, Stoler MH, Vibat CRT, et al: Performance and diagnostic accuracy of a urine-based human papillomavirus assay in a referral population. Cancer Epidemiol Biomarkers Prev 26: 1053-1059, 2017

72. Torres-Ibarra L, Lazcano-Ponce E, Franco EL, Cuzick J, Hernandez-Avila M, Lorincz A, Rivera B, Ramírez P, Mendiola-Pastrana I, Rudolph S, et al: Triage strategies in cervical cancer detection in Mexico: Methods of the FRIDA study. Salud Publica Mex 58: 197-210, 2016.

73. Torres-Ibarra L, Cuzick J, Lorincz AT, Spiegelman D, Lazcano-Ponce E, Franco E, Moscicki A, Mahmud S, Wheeler C, Rivera-Paredez B, et al: Comparison of HPV-16 and HPV-18 genotyping and cytological testing as triage testing within human papillomavirus-based screening in Mexico. JAMA Netw Open 2: e1915781, 2019.

74. Cuzick J, Du R, Adcock R, Kinney W, Joste N, McDonald R, English K, Torres S, Saslow D and Wheeler C; New Mexico HPV Pap Registry Steering Committee: Uptake of co-testing with HPV and cytology for cervical screening: A population-based evaluation in the United States. Gynecol Oncol 162: 555-559, 2021.

75. Kyrgiou M, Arbyn M, Bergeron C, Bosch F, Dillner J, Jit M Kim J, Poljak M, Nieminen P, Sasieni P, et al: Cervical screening: ESGO-EFC position paper of the European Society of Gynaecologic Oncology (ESGO) and the European Federation of Colposcopy (EFC). Br J Cancer 123: 510-517, 2020

76. Castle PE, Adcock R, Cuzick J, Wentzensen N, Torrez-Martinez N, Torres S, Stoler M, Ronnett B, Joste N, Darragh T, et al: Relationships of p16 immunohistochemistry and other biomarkers with diagnoses of cervical abnormalities: Implications for LAST terminology. Arch Pathol Lab Med 144: 725-734, 2019.

77. Macedo AC, Gonçalves JC, Bavaresco DV, Grande AJ, Chiaramonte Silva N and Rosa MI: Accuracy of mRNA HPV tests for triage of precursor lesions and cervical cancer: A systematic review and meta-analysis. J Oncol 2019: 6935030, 2019.

78. Shah UJ, Nasiruddin M, Dar SA, Khan MK, Akhter MR, Singh N, Rabaan AA and Haque S: Emerging biomarkers and clinical significance of HPV genotyping in prevention and management of Cervical Cancer. Microb Pathog 143: 104131, 2020.
79. van Leeuwen RW, Oštrbenk A, Poljak M, van der Zee AG, Schuuring E and Wisman GB: DNA methylation markers as a triage test for identification of cervical lesions in a high risk human papillomavirus positive screening cohort. Int J Cancer 144: 746-754, 2018.

80. Tian Y, Yuan Wu NY, Liou YL, Yeh CT, Cao L, Kang YN, Wang HJ, Li Y, Chu TY, Li W, et al: Utility of gene methylation analysis, cytological examination, and HPV-16/18 genotyping in triage of high-risk human papilloma virus-positive women. Oncotarget 8: 62274-62285, 2017.

81. Banila C, Lorincz AT, Scibior-Bentkowska D, Clifford GM, Kumbi B, Beyene D, Wheeler CM, Cuschieri K, Cuzick J and Nedjai B: Clinical performance of methylation as a biomarker for cervical carcinoma in situ and cancer diagnosis: A worldwide study. Int J Cancer 150: 290-302, 2021.

82. Cook DA, Krajden M, Brentnall AR, Krajden M, Gondara L, Chan T, Law J, Smith L, Niekerk D, Ogilvie G, et al: Evaluation of a validated methylation triage signature for human papillomavirus positive women in the HPV FOCAL cervical cancer screening trial. Int J Cancer 144: 2587-2595, 2018

83. Louvanto K, Franco EL, Ramanakumar AV, Vasiljević N, Scibior-Bentkowska D, Koushik A, Cuzick J, Coutlée F and Lorincz AT; Biomarkers of Cervical Cancer Risk Study Team: Methylation of viral and host genes and severity of cervical lesions associated with human papillomavirus type 16. Int J Cancer 136: E638-E645, 2015.

84. Vale D, Teixeira J, Bragança J, Derchain S, Sarian L and Zeferino L: Elimination of cervical cancer in low- and middle-income countries: Inequality of access and fragile healthcare systems. Int J Gynecol Obstet 152: 7-11, 2020.

85. Ngwalle EW, Mgaya HN, Mpanju-Shumbusho W, Chirenje ZM, Kirumbi L, Lebelle T and Kaggwa S: Situation analysis for diagnosis and treatment of cervical cancer in mainland Tanzania. East Afr Med J 78: 60-64, 2001.

86. Rosser JI, Hamisi S, Njoroge B and Huchko MJ: Barriers to cervical cancer screening in rural Kenya: Perspectives from a provider survey. J Community Health 40: 756-761, 2015.

87. McDonald YJ, Goldberg DW, Scarinci IC, Castle PE, Cuzick J, Robertson M and Wheeler CM: Health Service Accessibility and risk in cervical cancer prevention: Comparing rural versus nonrural residence in New Mexico. J Rural Health 33: 382-392, 2017.

88. Bingham A, Bishop A, Coffey P, Winkler J, Bradley J, Dzuba I and Agurto I: Factors affecting utilization of cervical cancer prevention services in low-resource settings. Salud Publica Mex 45 (Suppl 3): S408-S416, 2003.

89. Markovic M, Kesic V, Topic L and Matejic B: Barriers to cervical cancer screening: A qualitative study with women in Serbia. Soc Sci Med 61: 2528-2535, 2005

90. Urasa M and Darj E: Knowledge of cervical cancer and screening practices of nurses at a regional hospital in Tanzania. Afr Health Sci 11: 48-57, 2011.

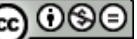

This work is licensed under a Creative Commons

Attribution-NonCommercial-NoDerivatives 4.0 International (CC BY-NC-ND 4.0) License. 